\title{
Early Detection of Steatohepatitis: Less Teeth NASHing and More Research?
}

\author{
Emmanuel Thomas ${ }^{1} \cdot$ Eugene R. Schiff ${ }^{2}$
}

Published online: 1 June 2016

(c) Springer Science+Business Media New York 2016

The prevalence of nonalcoholic steatohepatitis (NASH) is increasing rapidly; particularly, alarming is the rising incidence among children [1]. Importantly, NASH is becoming a leading cause of hepatic decompensation that necessitates liver transplant. Despite its mounting occurrence and increasing burden on the healthcare system, NASH is underdiagnosed in clinical practice, even among hepatologists and gastroenterologists, due to the lack of specific biomarkers and imaging studies that facilitate its early diagnosis. This is a major hurdle clinically since the earlier the recognition of NASH, the greater the chance of limiting disease progression and facilitating a cure. In order to diagnose NASH earlier, there is a need for identifying biomarkers of NASH, differentiating it from fatty metamorphosis alone although recent findings may aid in this endeavor [2].

To address disease progression from a mechanistic standpoint, differences in wound-healing responses to lipotoxic hepatocytes may differentiate the progressive forms of NASH from reversible disease [3, 4]. Therefore, well-compensated cirrhosis in NASH patients is potentially reversible if the underlying necroinflammatory process is stopped prior to hepatic decompensation. Illustrating the potential reversibility of early pathological changes in liver

Eugene R. Schiff

eschiff@med.miami.edu

$1 \quad$ Schiff Center for Liver Diseases and Sylvester Cancer Center, University of Miami Miller School of Medicine, Miami, FL, USA

2 Leonard Miller Professor of Medicine, Dr. Nasser Ibrahim Al-Rashid Chair in the Schiff Center for Liver Diseases, University of Miami Miller School of Medicine, Jackson Medical Tower, 1500 N.W. 12 Ave, E-1101, Miami, FL 33136, USA diseases, successful therapy for viral hepatitis, immunosuppressive therapy for autoimmune hepatitis, and complete abstinence for alcohol intake have all facilitated fibrosis regression.

Currently, with respect to assessing the presence and severity of NASH, liver biopsy is the most definitive diagnostic test. Even though biopsy is required for most clinical trials, it is not performed in most non-study patients [5]. Less invasive modalities that detect fatty metamorphosis and fibrosis are becoming widely available. Two relatively new diagnostic tools that are currently being utilized include the performance of assessment utilizing a Fibroscan ${ }^{\circledR}$ with controlled attenuation parameter (CAP) module and also magnetic resonance elastography (MRE).

With regard to therapy for existing disease, a variety of new clinical trials assessing the safety and efficacy of potential drugs that reduce the severity of NASH and facilitate disease regression are underway [6]. Nevertheless, as a potential treatment for NASH, an oral drug that safely and effectively produces involuntary weight loss has yet to be developed. Yet, this type of therapeutic approach circumvents the fundamental challenge of changing the habits that culminate in the metabolic syndrome, which is characterized by diabetes, hypertension, hyperlipidemia, and obesity, all comorbid conditions that persist even if $\mathrm{NASH}$ regresses [7]. NASH-targeted drugs are being utilized to slow the progression of steatohepatitis with resolution of hepatocytic ballooning, necroinflammation, Mallory bodies when present, and steatosis. Most important will be the reproducible facilitation of regression of fibrosis and a subsequent reduction of the risk of hepatocellular carcinoma, which has been observed for therapy with statins that are frequently used in NASH patients [8] although the underlying mechanisms remain to be clarified. Selective anti-inflammatory agents targeting cytokines and 
modification of specific immunologic pathways that are involved in disease progression are evolving [9].

In this issue of Digestive Diseases of Sciences, the manuscript by Nagpal et al. [10] underscores the insidious nature of nonalcoholic steatohepatitis (NASH). The investigators specifically collected data on patients on their knowledge of their underlying liver disease and existing clinical manifestations at their initial transplant evaluation visit. The major finding was that advanced cirrhosis and hepatic decompensation, as indicated by the presence of ascites, were already present prior to the recognition of NASH in the majority of patients that were being evaluated for liver transplant. This late diagnosis and presentation with advanced disease would likely impair attempts to reduce disease severity through the institution of lifestyle changes and drugs, underscoring importance of early detection. In concordance with these findings, many of these patients did not undergo a liver biopsy to assess their liver disease prior to referral for transplant. Furthermore, most patients did not receive any therapy to prevent NASH-related disease progression.

Overall, the study illustrates the need for the broader implementation of screening guidelines to identify NASH patients well before advanced disease has developed. One particularly interesting finding was that the diagnosis of hypercholesterolemia was associated with the earlier diagnosis of NASH. Indeed, the earlier diagnosis of NASH promoted patients to be evaluated for liver transplant with lower Mayo End-stage Liver Disease (MELD) scores and with decreased encephalopathy at presentation [10].

Limitations of the study include its single-site design and the relatively small number of study patients enrolled, limiting the power to achieve statistically significant conclusions for several of the comparisons. Moreover, there were no data presented on whether or not the patients had undergone evaluation by a hepatologist or gastroenterologist before being referred for liver transplant; furthermore, data were not presented correlating disease self-knowledge with the time length between specialist consultation and liver transplant evaluation. Indeed, these limitations may have resulted from the retrospective chart review study design as opposed to a prospective study of patients undergoing liver transplant evaluation that would involve direct patient data acquisition. Lastly, the study was conducted from 2006 to 2011 when the assessment of NASH was rudimentary compared with current diagnostic capabilities. Additional studies will be needed to confirm these data that support the initial diagnosis of NASH occurring at later stages of the natural history of the disease.

In conclusion, with the clinical development of many new agents to prevent NASH [6] and with improved methods of noninvasive assessment of liver fibrosis and steatosis [5], this study provides additional impetus to ramp up screening efforts. The authors acknowledge that although widespread screening efforts may be initially unattainable, more efforts focused on high-risk populations, including those with type 2 diabetes mellitus, would be a more feasible goal. It also alludes to the need for the increased awareness of NASH among family medicine practitioners, internists, cardiologists, and endocrinologists. The earlier referral of these "high risk" patients to a gastroenterologist/hepatologist would facilitate the implementation of preventive measures such as the administration of ursodeoxycholic acid or vitamin $\mathrm{E}$, when appropriate, coupled with lifestyle changes that facilitate weight loss through exercise and appropriate caloric and nutritional consumption. Most importantly, the approval of future successful therapies for those who already have NASH will unequivocally benefit the population at large, which is a worthy goal that will likely take many years to achieve.

\section{References}

1. Younossi ZM, Koenig AB, Abdelatif D, Fazel Y, Henry L, Wymer M. Global epidemiology of non-alcoholic fatty liver disease-meta-analytic assessment of prevalence, incidence and outcomes. Hepatology. 2015. doi:10.1002/hep.28431.

2. Romeo S, et al. Genetic variation in PNPLA3 confers susceptibility to nonalcoholic fatty liver disease. Nat Genet. 2008:40:1461-1465.

3. Machado MV, Diehl AM. Pathogenesis of nonalcoholic steatohepatitis. Gastroenterology. 2016. doi:10.1053/j.gastro.2016.02. 066.

4. Hirsova $P$, et al. Lipid-induced signaling causes release of inflammatory extracellular vesicles from hepatocytes. Gastroenterology. 2016;150:956-967.

5. Rinella ME, et al. Practice patterns in NAFLD and NASH: real life differs from published guidelines. Ther Adv Gastroenterol. 2016;9:4-12.

6. Lassailly G, Caiazzo R, Pattou F, Mathurin P. Perspectives on treatment for nonalcoholic steatohepatitis. Gastroenterology. 2016. doi:10.1053/j.gastro.2016.03.004.

7. Van Wagner LB, Serper M, Kang R, et al. Factors associated with major adverse cardiovascular events after liver transplantation among a national sample. Am J Transplant. 2016. doi:10.1111/ ajt.13779.

8. Nielsen SF, Nordestgaard BG, Bojesen SE. Statin use and reduced cancer-related mortality. $N$ Engl $J$ Med. 2012;367:1792-1802.

9. Tilg H, Moschen AR, Szabo G. Interleukin-1 and inflammasomes in ALD/AAH and NAFLD/NASH. Hepatology. 2016. doi:10. 1002/hep.28456.

10. Nagpal SJ, Kabbany MN, Mohamad B, Lopez R, Zein NN, Alkhouri N. Portal hypertension complications are frequently the first presentation of NAFLD in patients undergoing liver transplantation evaluation. Dig Dis Sci. (Epub ahead of print). doi:10. 1007/s10620-016-4124-3. 